\title{
VENTAJAS Y RIESGOS DE LA UTIUZACIÓN DEL ANTÍGENO PROSTÁTICO ESPECÍFICO (PSA) EN EL ÁREA SANITARIA V DE GIJÓN (ASTURIAS).
}

\author{
Javier Cepeda Piorno ${ }^{1}$, Manuel Rivas del Fresno², Eduardo Fuente Martín ${ }^{3}$, Esther G onzález \\ G arcía ${ }^{4}$, Valentín M uruamendiaraz Fernández ${ }^{2}$ y Eloy Fernández Rodríguez ${ }^{1}$.
}

Servicio de Análisis Clínicos ${ }^{1}$. Servicio de Urología ${ }^{2}$. Servicio de Anatomía Patológica ${ }^{3}$ y Servicio de Hematología ${ }^{4}$, Hospital de Cabueñes. G ijón. Asturias. España

\begin{abstract}
Resumen.- O BJETIVO: Con la introducción del PSA en la práctica clínica diaria, el diagnóstico del cáncer de próstata ha sufrido importantes cambios. Aunque el screening es controvertido, su uso parece que se ha generalizado. Conocer la utilización que se hace del PSA en nuestro área sanitaria y analizar los beneficios y riesgos que se perciben.

MÉTO DO: A través del archivo informático, analizamos las determinaciones realizadas durante los años 2000 y 2001 en nuestro área (290.956 habitantes). Se analizaron también las biopsias de próstata generadas y los cánceres detectados.
\end{abstract}

RESULTADO S: Se realizaron 25.590 determinaciones de PSA. Proceden de A tención Primaria 59\%, Urología

Fco. Javier Cepeda Piorno

Laboratorio de Análisis C línicos.

Hospital de Cabueñes.

C/ De los Prados, N 0395.

33203 G ijón. Asturias. (España)

J_cepeda_p@hotmail.com

Trabajo recibido: 28 de enero 2005
$34 \%$ y resto de especializada $7 \%$. El $39 \%$ se realizan a hombres mayores de 70 años. El PSA fue normal en el $78,7 \%$, y mayor de $4 \mathrm{ng} / \mathrm{mL}$ en $21,2 \%$. Se realizaron 488 biopsias prostáticas, detectándose 178 carcinomas $(36,5 \%$ rendimiento diagnóstico). Según el primer PSA el diagnóstico parte de primaria en $44 \%$, Urología en $46 \%$ y resto de especializada $10 \%$. El tiempo medio desde el primer PSA hasta el diagnóstico fue de 5 meses, sin diferencias significativas entre Primaria y Especializada. El uso del PSA por Primaria es variable (entre 8,1 y 45,8 determinaciones por cada 100 hombres mayores de 50 años), sin diferencias significativas de detección de cáncer prostático según número de PSAs ni diferencias de edad. Frente al periodo 1982-1993 la incidencia de cáncer prostático pasa de 30,76 a 52,8 nuevos casos/ 100.000 habitantes/ año. Existe mayor incidencia e incremento del cáncer en la zona rural (de 33,52 a 221,1 nuevos casos/ 100.000 habitantes/ años).

CON C LUSIO N ES: C onfirmamos la utilización generalizada de esta prueba y la tendencia al cribado en primaria, que participa de manera importante en el diagnóstico. El PSA adelanta el diagnóstico del cáncer de próstata en 5 años en nuestro área, y dispara las tasas de incidencia de este cáncer. La elevada utilización de este marcador en población de edad avanzada se puede considerar inadecuada.

Palabras clave: Antígeno prostático específico (PSA). Cáncer de próstata. Screening. 
Summary.- O BJEC TIVES: The diagnosis of prostate cancer has changed significantly with the introduction of PSA in the clinical practice. Despite screening is under controversy the use of PSA has become widespread. The objective of this paper is to know the use of PSA in our health-care area and to analyze perceived risks and benefits.

METHODS: From the informatic archives we analyze PSA determinations performed in our health-care area (290.956 citizens) over 2000 and 2001. We also analyzed prostate biopsies generated and number of cancers detected.

RESULTS: 25.519 PSA determinations were performed. $59 \%$ came from general practitioners (GP), $34 \%$ from urologists and $7 \%$ from the rest of specialists. $39 \%$ are performed to men older than 70 years. PSA was normal in $78.7 \%$ of the patients and higher than $4 \mathrm{ng} / \mathrm{ml}$ in $21.2 \%$. 488 prostatic biopsies were performed diagnosing 178 cancers (diagnostic yield $36.5 \%$ ). Depending on the first PSA, diagnosis was started by a $G P$ in $44 \%$ of the cases, a urologist in $46 \%$, and the remaining $10 \%$ by other specialists. Mean time from first PSA to diagnosis was 5 months, without significant differences between $G P s$ and specialities. The use of PSA by GPs is variable (between 8.1 and 45.8 determinations per 100 men over 50 years), without significant differences in prostate cancer detection by number of PSAs or differences in age. In comparison with the period 1982-1993 the incidence of prostate cancer goes from 30.76 to 52.8 new cases/ 100.000 inhabitants/year. There is a greater incidence and increase of cancer in the rural area (from 33.52 to 221.1 new cases/ 100.000 inhabitants/ year).

CONCLUSIONS: We confirm the general use of this test and the trend to screening in the primary health-care level, which participates in an important manner in the diagnosis. PSA brings forward the diagnosis of prostate cancer 5 years in our area, and shoots its incidence rates. The high use of such marker in our population of advanced age may be considered inadequate.

Keywords: PSA. Prostate cancer. Screening.

\section{INTRODUCCIÓN}

El carcinoma prostático (CAP) es un auténtico problema sanitario. En el hombre, el cáncer de próstata es el más frecuente del aparato genitourinario. Representa la tercera causa de muerte por cáncer después del de pulmón y colo-rectal, y la segunda causa de mortalidad en los mayores de 75 años (1).
Dada la sensibilidad del análisis inmunológico de detección del antígeno prostático específico (PSA), actualmente es posible una mayor precocidad en la detección de la patología prostática; como consecuencia, el diagnóstico del cáncer de próstata ha sufrido cambios significativos (2) y el uso clínico del PSA se ha generalizado.

Sin embargo, no existen evidencias clínicas definitivas sobre los beneficios del cribado sistemático de la población mediante PSA y tacto rectal (3).

Tampoco se ha demostrado que dicho cribado y el aumento en incidencia del cáncer prostático que comporta se haya traducido en una reducción en la mortalidad por este cáncer 0 en una mejora de la calidad de vida de los pacientes diagnosticados (4). También está por aclarar la proporción de neoplasias de próstata diagnosticadas gracias a la utilización del PSA, que serían clínicamente no significativas (4).

Por todo ello nos interesa analizar el uso que se hace del PSA en nuestro área sanitaria, así como los beneficios y riesgos de su utilización.

\section{MATERIAL Y MÉTODO}

El estudio se realiza en el Hospital de Cabueñes de Gijón (A sturias), que es el Hospital de referencia del área sanitaria $V$ y atiende a los municipios de Gijón, Carreño y Villaviciosa, de carácter fundamentalmente mixto (urbano y rural). Da cobertura a 290.956 habitantes (138.470 hombres y 152.482 mujeres) de los cuales 51.771 son hombres mayores de 50 años (el 17,79\% de la población general). $\mathrm{N}$ uestra investigación se centró en este grupo de población. El diseño del estudio es longitudinal retrospectivo durante un periodo de dos años (2000 y 2001).

Se recogieron los datos correspondientes a las determinaciones de PSA, a través de la base de datos informática de nuestro laboratorio. Las variables que utilizamos fueron: fecha de la petición, médico y servicio peticionario, edad del paciente y resultado de la determinación. También se recogieron el número de historia clínica y los nombres y a pellidos de los pacientes para las comprobaciones necesarias. La edad no se refleja en todos los volantes de petición, con lo que 
en los cálculos que incluimos la edad trabajamos sólo con aquellos en los que se reflejaba este dato, y tomando en cuenta las edades que nos interesaban: entre 50 y 70 años y mayores de 70 años. Se eliminaron todas las determinaciones incorrectas (mujeres, niños) o con edades no coherentes, posiblemente por error en la solicitud de la petición o por errores de transcripción a la hora de introducir los datos en el ordenador.

Los resultados de las biopsias de próstata se obtuvieron seleccionando exclusivamente las biopsias por punción (normalmente biopsias bilaterales de próstata o cilindros prostáticos mediante ecografía transrectal), que serían las realizadas probablemente por un PSA alterado y rechazamos las correspondientes a resecciones transuretrales de próstata, adenectomías, o de muestras procedentes de hiperplasias benignas de próstata, ya que estos hallazgos no serían motivados por la utilización del PSA. Para completar el estudio de las biopsias del área sanitaria $V$ nos faltarían las correspondientes a otro hospital, que agrupa dos grandes centros de salud que no pudimos incluir en el estudio, puesto que se nos denegaron los datos solicitados.

Una vez identificados los carcinomas, revisamos en la base de datos informática si tenían peticiones de PSA previas, o revisamos la historia clínica cuando fue necesario. No incluimos en el estudio los CAP sin determinaciones de PSA previas a la biopsia o posteriores a ella.

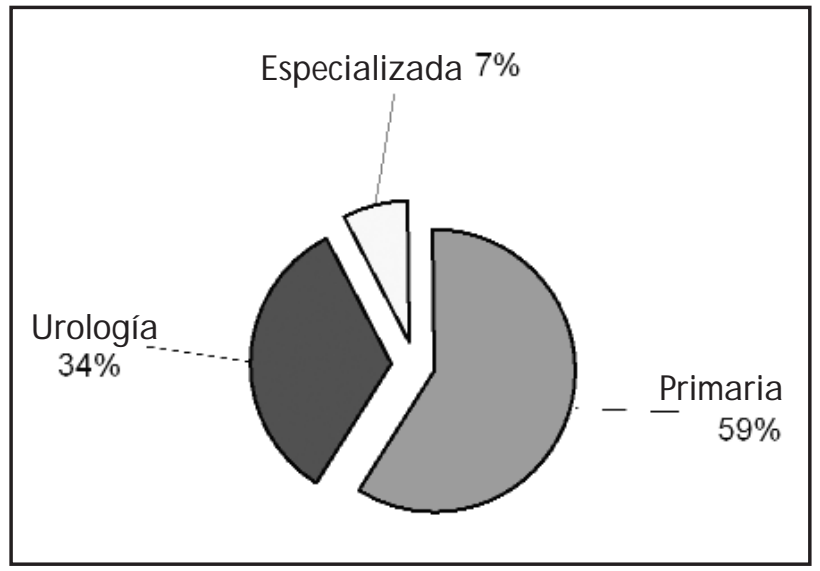

FIG URA 1. Distribución de las peticiones de PSA, según el origen de la petición.
Para conocer cuántos PSA se utilizan para cada CAP detectado se recurre a la historia clínica, si con la base de datos informática no es suficiente 0 si existen dudas sobre las determinaciones o la identidad del paciente. Sólo tomamos en cuenta las determinaciones realizadas por nuestro laboratorio y no las realizadas por otros laboratorios públicos o privados.

La detección del cáncer se la adjudicamos al servicio al que pertenece el facultativo que solicitó el primer PSA, que entendemos que fue el primero en comenzar la investigación que finalmente concluyó con el descubrimiento del carcinoma. A sí los agrupamos en: procedentes de Primaria, U rología, o del resto de Servicios de Especializada.

Para el análisis estadístico utilizamos distribuciones de frecuencia, media o mediana dependiendo del tipo de distribución: paramétrica o no paramétrica. Para ello se utilizó el Wilcoxon test a través del programa informático Medcal (versión 6.14).

Los cálculos de las tasas de incidencia se realizaron con el programa esta dístico Epidat (versión 2.1 para windows) y para el ajuste de tasas por el método directo se empleó la población estándar Europea (5).

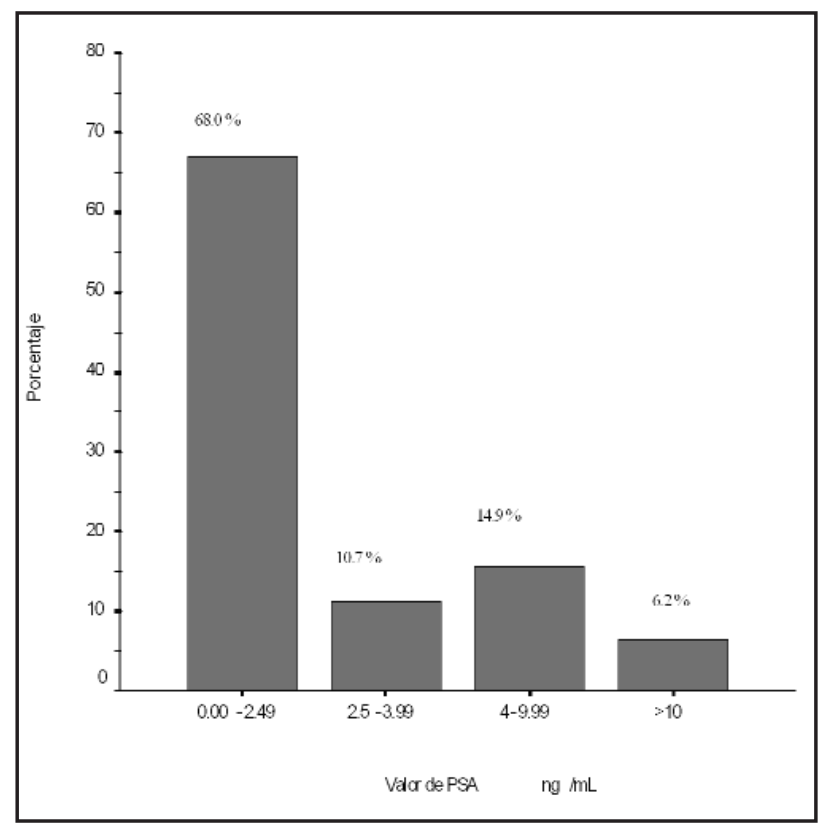

FIG URA 2. Distribución de las peticiones de PSA realizadas, según el valor del PSA obtenido. 


\section{RESULTADOS}

Durante el período 2000-2001 se realizan en nuestro hospital 25.590 determinaciones de PSA. El origen peticionario es primaria en 15.014 determinaciones (59\% del total), urología en 8.659 determinaciones ( $34 \%$ del total) y el resto de servicios de especializada en 1.917 determinaciones (7 \% del total). Los resultados se representan en la Figura 1.

Los servicios de especializada que solicitaron algún PSA fueron: Medicina Interna, Digestivo, Hematología, Nefrología, Cardiología, Cirugía, Dermatología, Endocrino, Hemodiálisis, Medicina Preventiva, Neumología, O ncología, Traumatología, N eurología, 0 torrinolaringología y Reumatología.

Para estudiar las determinaciones realizadas por tramos de edades nos quedamos con 12.216 peticiones, que son las que tienen este dato y cumplen con los criterios de inclusión. El $61 \%$ de estas determinaciones se realizan a hombres entre 50 y 70 años (que en porcentaje ocupan el $70 \%$ de la población mayor de 50 años) y el $39 \%$ de las peticiones se realizan a pacientes mayores de 70 años (que ocupan el $30 \%$ de la población mayor de 50 años).

El punto de corte para el nivel de PSA sérico en nuestro laboratorio está en $4 \mathrm{ng} / \mathrm{mL}$. De las 25.590 determinaciones realizadas, 5409 tuvieron un valor mayor 0 igual a $4 \mathrm{ng} / \mathrm{mL}$ (el $21,1 \%$ de todas las determinaciones). Tuvimos 1.584 determinaciones que dieron un PSA $10 \mathrm{ng} / \mathrm{mL}$ (el $6,2 \%$ de las determinaciones realizadas). La distribución de las deter-

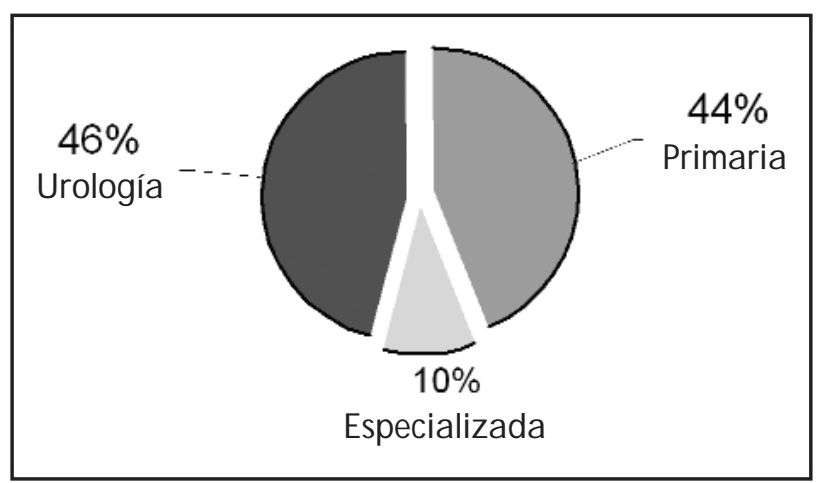

FIG URA 3. Distribución de los CAP según el responsable del diagnóstico. minaciones según el valor del PSA se representa en la Figura 2.

Durante este período de tiempo se enviaron al laboratorio de anatomía patológica 488 biopsias, procedentes tanto del servicio de Urología como de Radiología. Del total de biopsias realizadas se detectó CA P en 178 pacientes (el $36,5 \%$ de todas las biopsias realizadas, lo que correspondería con nuestro rendimiento diagnóstico).

De estos CA P detectados, la solicitud del primer PSA fue realizada por primaria en el $44 \%$ de los casos (en 78 pacientes), urología en el $46 \%$ de los casos (en 82 pacientes) y por el resto de servicios de especializada en el $10 \%$ restante (en 18 pacientes). Los resultados se representan en la Figura 3.

Los servicios de especializada que detectaron algún CAP fueron: M edicina interna (10), N eumología (2), Traumatología (2), Cirugía (2), Digestivo (1) y Endocrino (1).

El tiempo que se tardó en diagnosticar un CAP desde la realización del primer PSA hasta el resultado positivo de la biopsia fue de 5 meses (154 días; 98,6-336,6; IC 95 \%). Primaria tardó 3,9 meses (117 días; 81,9-290; IC $95 \%$ ) mientras que Especializada tardó 6,9 meses (207,5 días; 94,3546,2 ; IC $95 \%$ ) para el diagnóstico del CAP. Sin embargo, por la gran variación en los resultados, estas diferencias no son estadísticamente significativas ( $p>0,1$, W ilcoxon test). Los resultados se representan en la Figura 4.

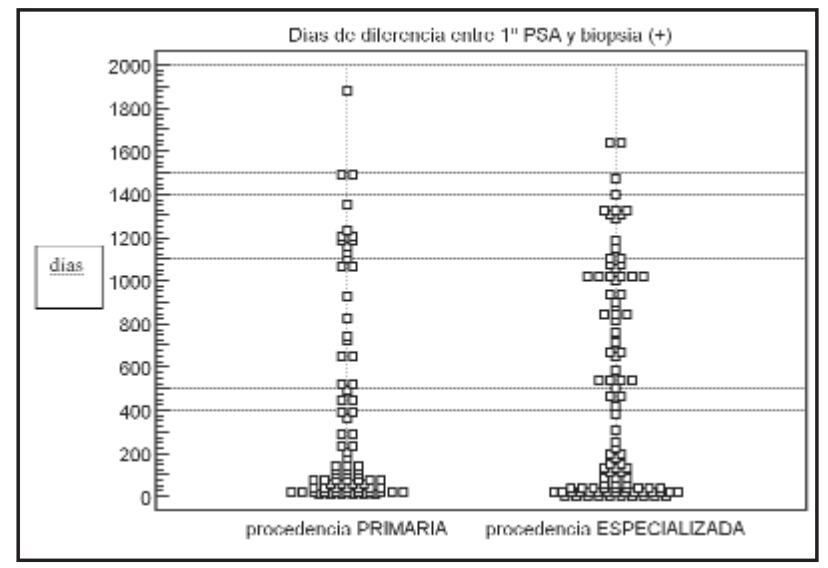

FIG URA 4. Tiempo que se tarda en diagnosticar un CAP con el PSA desde primaria y especializada. 
En el $66 \%$ de los CAP detectados se utilizaron entre 1 y 2 determinaciones de PSA. En el resto se utilizaron más de 2 determinaciones. En un $38 \%$ de los diagnósticos (69 pacientes), se utilizó un único PSA: 28 pacientes procedían de primaria, 8 de especializada y 33 pacientes de urología. Los resultados se representan en la Figura 5.

A nalizando la utilización del PSA por los distintos ambulatorios de nuestra área sanitaria obtenemos los resultados que se indican en la Tabla I.

O bservamos que existen diferencias en cuanto a la utilización del PSA en los distintos ambulatorios. En algunos centros existe una gran demanda de esta prueba, mientras que en otros la demanda es mucho menor. Los casos extremos son los centros de salud de Contrueces y Los Campos: mientras el primero realiza 8,1 peticiones de PSA por cada 100 hombres mayores de 50 años, el segundo realiza 45,8 determinaciones.

Estos datos los intentamos correlacionar con las tasas de detección obtenidas en los distintos centros de salud, pero no encontramos diferencias estadísticamente significativas en cuanto a la detección, ni correlación alguna. Tampoco encontramos asociación entre la mediana de edad en el diagnóstico y las tasas encontradas.

Para conocer la incidencia de CAP en estos dos años, distinguimos entre el área rural (Villaviciosa) y el área urbana (G ijón). En la era pre-PSA (que vamos a considerarla desde el año 1982 al 1993, puesto que son los datos que nos ofrece el libro blanco del cáncer en A sturias) (6), se detectaron en G ijón 443 casos, con una tasa estandarizada por el método directo a la población estándar europea: TED 30,76 casos / 100.000 hombres (EE 1,47), mientras que en Villaviciosa se detectaron 54 casos, con una TED 33,52 casos/ 100.000 hombres (EE 4,61). Hay que tener en cuenta que estos datos corresponden a carcinomas detectados tanto clínica como histológicamente. Aún así, nos sirven a modo orientativo para compararlos con nuestros datos actuales. En estos años, el porcentaje de variación anual de cambio en la incidencia era de - $3 \%$ (es decir, la incidencia de este cáncer, se encontraba en situación descendente). La mediana de edad en el diagnóstico era de 75 años (6).

Durante los años 2000 y 2001, y sólo con la utilización del PSA según nuestro estudio, se obtiene una tasa estandarizada por el método directo a la población estándar Europea: TED en Gijón de 52,8 casos/ 100.000 hombres, mientras que en Villaviciosa se llegó a una TED 221,1 casos/ 100.000 hombres. La media de edad de los pacientes diagnosticados con el PSA fue de 69,86 años $(68,7-71,01$; IC $95 \%)$; mientras que la mediana de edad fue de 70 años (69-72 años; IC $95 \%$ ).

La distribución de las edades de los pacientes a la fecha del diagnóstico del CAP mediante la utilización del PSA se representa en la Figura 6.

TABLA I. UTIUZACIÓN DEL PSA POR LO S DISTIN TO S AM BULATO RIO S DEL ÁREA SAN ITARIA V DE GIJÓN.

\begin{tabular}{|l|c|c|c|c|c|c|}
\hline A M BULATO RIO S & $\begin{array}{c}\text { HO M BRES } \\
>50 \text { AN OS }\end{array}$ & $\begin{array}{c}\text { PETICIO N ES } \\
\text { PSA }\end{array}$ & $\begin{array}{c}\text { PSA/ 100 } \\
\text { HO M BRES }\end{array}$ & $\begin{array}{c}\text { CAP } \\
\text { DETECTA D O S }\end{array}$ & $\begin{array}{c}\text { TASAS BRUTA S } \\
\text { TRUN CADAS }\end{array}$ & EDA D (rango) \\
\hline Pumarín & 3443 & 940 & 27.3 & 3 & 87.1 & $76(63-78)$ \\
Severo O choa & 5825 & 1011 & 17.4 & 5 & 85.8 & $67(51-77)$ \\
Contrueces & 3436 & 279 & 8.1 & 3 & 87.3 & $68(64-69)$ \\
Laviada & 3339 & 1023 & 30.6 & 2 & 59.9 & $66(61-71)$ \\
Puerta de la Villa & 5084 & 1649 & 32.4 & 10 & 196.7 & $72(69-86)$ \\
Los Campos & 3739 & 1711 & 45.8 & 6 & 160.5 & $71(59-80)$ \\
El Llano & 5403 & 1321 & 24.4 & 14 & 259.1 & $69(53-85)$ \\
El Coto & 3492 & 869 & 24.9 & 9 & 257.7 & $71(60-78)$ \\
Parque Somio & 5322 & 1617 & 30.4 & 8 & 150.3 & $73(54-84)$ \\
Villaviciosa & 2530 & 990 & 39.1 & 14 & 553.4 & $71(50-80)$ \\
\hline
\end{tabular}




\section{DISCUSIÓN}

Se ha estimado que la detección de un cáncer prostático por alteración única del PSA viene a ser unos 6'2 años más precoz que la que se detectaría por la alteración del tacto prostático por vía rectal, y se acepta que estos tumores están más frecuentemente localizados en la glándula que los detectados a raíz de un tacto anómalo (7). Gracias a esta determinación analítica cualquier médico, sin necesidad de especial entrenamiento urológico, puede efectuar diagnósticos precoces del carcinoma prostático.

Sin embargo, los hipotéticos beneficios de esta actitud se ven diluidos por tres problemas inherentes al carcinoma prostático:

- la gran prevalencia de carcinoma prostático latente, que nunca se manifestaría en vida, por lo que su diagnóstico no va a aportar nada a la salud de su portador (e incluso le generará problemas de ansiedad y sobretratamiento).

- la lenta historia natural del carcinoma prostático, cifrada en años incluso para los carcinomas clínicamente significativos (8), por lo que no todos los pacientes (sólo los más jóvenes) van a tener tiempo para desarrollar la enfermedad clínicamente.

- la efectividad de los tratamientos no se conoce con certeza, puesto que la mayoría de los estudios dispo-

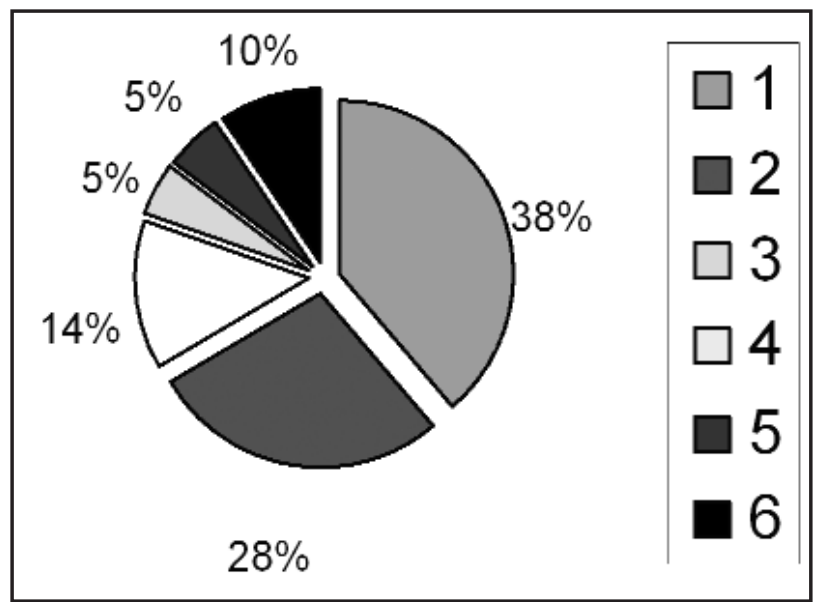

FIG URA 5. Determinaciones de PSA empleadas para los CAP detectados. nibles son observacionales, así que sus resultados deben de interpretarse con cautela (3). Si bien, es coincidente la opinión de optar por un tratamiento activo en la mayoría de casos, cada especialista se decanta por la técnica relacionada con su propia especialidad (9). A demás hay que tener en cuenta que la opción de tratamiento más ampliamente aceptada en pacientes ancianos (mayores de 70 años, o con una esperanza de vida inferior a 10 años), es la "espera vigilante", es decir, no tratamiento.

Aunque no existan suficientes datos epidemiológicos para estimar la prevalencia real del cáncer de próstata, los datos existentes sugieren que podría ser el más prevalente de todos los cánceres si se incluyen los casos detectados en autopsias y en biopsias de intervenidos por patología prostática benigna. La prevalencia del cáncer de próstata en autopsias de varones mayores de 50 años varía entre el $9 \%$ y el $46 \%$, llegando hasta el $90 \%$ a los 90 años (10). Esto hace sospechar que la mayoría de carcinomas prostáticos pasan inadvertidos en vida del paciente.

Por todo esto el cribado del carcinoma prostático mediante el uso sistemático del tacto rectal y el PSA es muy discutido. Así, no apoyan este cribado: U.S. Preventive Services Task Force (1996) (11), Red Internacional de Agencias de Evaluación de Tecnologías Sanitarias (IN HATA 1999) (3), Programa de Actividades Preventivas y de Promoción de la Salud (PAPPS) de la semFYC,(1999) (12), Health Services Utilization and Research Comisión de Canadá (2000), The NHS Prostate Cancer Programme (2000) (13), A merican College of Physicians, American Society of Internal Medice (3).

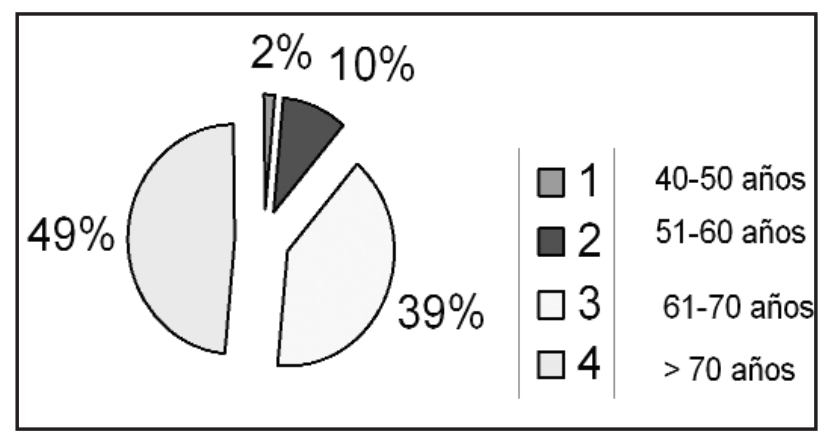

FIG URA 6. Edad de los pacientes a la fecha del diagnóstico del CAP utilizando el PSA. 
Por el contrario, sí apoyan el cribado del carcinoma prostático la A merican Cancer Society (3) y la A merican Urological Association (3). Pero en ambos casos (y esto creemos que es muy importante) siempre en personas con esperanza de vida superior a 10 años y previa información de los riesgos y beneficios.

Algunos estudios de análisis de decisiones han sugerido que la esperanza de vida disminuye con el cribado del carcinoma prostático si se tiene en cuenta la calidad de vida (14). El grupo de Krahn et al (15) demuestran que las pruebas de detección del carcinoma prostático pueden reducir de forma marginal la mortalidad por este cáncer, pero sugieren que este beneficio se ve superado de lejos por la morbilidad que el tratamiento genera. 0 tros análisis de decisión (16) han centrado su atención en los efectos del tratamiento, concluyendo que incrementa la esperanza de vida ajusta da a la calidad en menos de un año, disminuyéndola en aquellos pacientes de edad superior a 70 años y también en aquellos con carcinoma prostático bien diferenciado.

De nuestro estudio se desprende que la utilización del PSA parece generalizada, tanto desde primaria como desde especializada, pues prácticamente todos los servicios del hospital utilizan esta prueba.

La valoración clínica debería de ser distinta desde primaria o especializada, puesto que la prevalencia es distinta, lo que se refleja claramente al conocer las determinaciones realizadas en cada ámbito y los carcinoma prostático detectados: primaria realiza 192 determinaciones por cada CAP detectado, mientras que Urología realiza 105 y especializada 106 determinaciones por cada cáncer de próstata detectado.

La población que procede de primaria será mayoritariamente asintomática, lo que explicaría la menor detección de carcinoma prostático, mientras que la población de Urología o especializada corresponde con pacientes seleccionados, en los que la probabilidad de encontrar un cáncer de próstata es mayor.

Cuando analizamos las peticiones desde los distintos a mbulatorios, observamos distintos comportamientos dependiendo de los centros de salud. En algunos se observa una elevada demanda de peticiones con respecto a su población (hasta 45,8 determinaciones por cada 100 hombres mayores de 50 años).

Con estos datos parecen confirmarse nuestras sospechas, y se pone de manifiesto la clara tendencia que existe en primaria, hacia la realización de un cribado oportunista del CAP.

El distinto comportamiento a la hora de solicitar esta prueba no parece tener relación con la mayor o menor detección de cáncer, quizá por la menor prevalencia de esta enfermedad en el ámbito de primaria. La diferente manera de utilizar este marcador tumoral quizá se deba a que no existen guías claras de actuación, con lo que depende de la actitud que tenga cada facultativo con respecto al cribado, lo que va a motivar la mayor o menor demanda de esta prueba.

Un dato que nos llama la atención es el número elevado de cánceres que se detectan utilizando un único PSA (en 69 pacientes, del total de 178 diagnosticados). No parece que se utilice la prueba para seguimiento, como sería recomendable, sino más bien parecen determinaciones esporádicas que se realizan junto con analíticas de rutina. Según nuestra revisión, debido a la gran variabilidad interindividual que sufre este marcador en sus niveles séricos, utilizar una única determinación no debe considerarse una buena práctica:

- Un estudio realizado por Roerhban et al (17), en su serie de pacientes hasta el $55 \%$ tenían disminuidos los valores del marcador a los tres meses de la determinación inicial, un $40 \%$ tenían aumentos y sólo un $6 \%$

\section{TABLA II. VA LO RES DE N O RM AUDAD DEL PSA SEG ÚN LA EDAD DEL PACIEN TE. (SEG ÚN O ESTERUN G).}

\begin{tabular}{|l|}
\hline Entre $40-49$ años: $<2^{\prime} 5 \mathrm{ng} / \mathrm{mL}$ \\
\hline Entre $50-59$ años: $<3^{\prime} 5 \mathrm{ng} / \mathrm{mL}$ \\
\hline Entre $60-69$ años: $<4^{\prime} 5 \mathrm{ng} / \mathrm{mL}$ \\
\hline Entre $70-79$ años: $<6^{\prime} 5 \mathrm{ng} / \mathrm{mL}$ \\
\hline
\end{tabular}


mantuvo niveles idénticos en las dos determinaciones. A simismo, casi un tercio de los pacientes presentaron variaciones de $3 \mathrm{ng} / \mathrm{mL}$ en uno y otro sentido, hecho que implica que el $3,5 \%$ de los pacientes habrían cruzado el valor de referencia para ningún tipo de actitud terapéutica entre las dos determinaciones, con un riesgo de indicación incorrecta para el $10 \%$ de los pacientes.

- Se han podido constatar fluctuaciones fisiológicas en los niveles séricos de PSA; no son variaciones circadianas, más bien son variaciones al azar, aunque de pequeña intensidad (18).

- A intervalos de 4 horas durante un día completo, realizadas en pacientes con cáncer de próstata en estadío D2, en pacientes de HBP y en individuos sanos, se observan fluctuaciones a lo largo del día, con una variación media de $17,6+/-2,3$, sin diferencias entre los distintos grupos, por lo que se recomienda no confiar en una sola determinación (19).

A la vista de estos datos, consideramos que sería más razonable la realización como mínimo de 2 determinaciones de PSA, antes de realizar ninguna biopsia. Hay que tener en cuenta que también pueden ocurrir falsas elevaciones del PSA que habría que descartar, por ejemplo: en casos de prostatitis, infecciones urinarias, retenciones urinarias, eyaculaciones, cistoscopias, sondajes 0 actividades como montar en bicicleta que se han demostrado que alteran el PSA (20).

0 tro dato relevante que obtenemos es el gran número de peticiones de PSA que se realizan en ancianos (mayores de 70 años). Si bien el cribado en pacientes jóvenes (menores de 70 años o con esperanza de vida superior a 10 años) es controvertido, lo que no cabe duda es que en pacientes ancianos el cribado no aporta grandes beneficios, sino al contrario; puede acarrear importantes daños para el paciente, que además se traducen en una mayor demanda de servicios y en un incremento del gasto sanitario considerable, que se podría dedicar a otras actividades que produzcan beneficios netos comprobados, para la salud individual y pública.

A este respecto, algunos autores proponen utilizar límites de normalidad del PSA considerando intervalos de edad (21).(Ver Tabla II). Utilizando estos límites edad-específicos se podrían diagnosticar el
$18 \%$ más de neoplasias en varones menores de 60 años y se perderían el $4-22 \%$ de neoplasias en los mayores de 60 años (22). Por ahora, los límites por intervalos de edad no son recomendados institucionalmente, pero a nuestro entender, podrían hacer reflexionar al clínico sobre la necesidad de interpretar el resultado analítico dentro del contexto clínico determinado, y muy importante, teniendo en cuenta la edad del paciente.

Como se comentó, en nuestro área sanitaria se realizaron 25.590 determinaciones de PSA, con 5409 resultados patológicos, descubriéndose 178 carcinomas prostáticos. De ellos, los que se podrían beneficiar teóricamente del tratamiento curativo (los menores de 70 años) serían 81 pacientes (el 16\% de los biopsiados), lo que parece una proporción muy pequeña respecto al número total de pacientes estudiados. Pocos se van a beneficiar del tratamiento curativo, mientras que muchos van a sufrir sus efectos secundarios, sin saber realmente el efecto beneficioso que les está aportando.

Como efecto positivo del uso del PSA, vemos que el diagnóstico del CAP se ha adelantado 5 años con respecto a la época en la que no se utilizaba esta prueba. A ún así, la mediana de edad está en 70 años, con lo que considerar que se está realizando un diagnóstico precoz parece un poco alejado de la realidad. $\mathrm{N}$ uestro estudio tiene las limitaciones que se le pueden atribuir a todo diseño descriptivo. Además nuestra fuente de datos informática puede contener algún error debido a la transcripción de los datos referidos a las edades de los pacientes, por ello eliminamos gran número de determinaciones para poder trabajar con las edades, que nos parecía un dato importante. Aún así, no pensamos que se produzcan grandes sesgos en la selección del grupo de estudio.

O tra limitación fue tener que trabajar sólo con determinaciones realizadas y no con número de pacientes, ya que al no coincidir el número de historia de primaria y especializada es imposible afinar en este dato y el número exacto de pacientes analizados no lo conocemos. De esta manera no podemos realizar cálculos de sensibilidad, especificidad o tasas de detección.

De todos modos con los datos obtenidos ha sido suficiente para cumplir con los objetivos de nues- 
tro estudio, que más que obtener datos exactos, pretendíamos conocer de modo general la utilización del PSA en nuestra área sanitaria y su repercusión en el diagnóstico del CAP.

Pretendíamos hacer una fotografía de la realidad de nuestro medio, y a partir de aquí tomar las medidas correctoras oportunas para racionalizar el uso de ésta prueba diagnóstica, que tiene una importante repercusión social, repercusión en el propio sistema sanitario en general y en los individuos en particular.

Agradecimientos: Quisiera expresar mi más sincero a gradecimiento a todo el Servicio de Análisis Clínicos del Hospital de Cabueñes, por su apoyo incondicional en todo momento y por su gran profesionalidad.

\section{BIBUOGRAFIA y LECTURAS RECOMENDADAS (*lectura de interés y **lectura fundamental)}

1. WESTRA, C.S.; DUCATMAN, A.M.; NIEWIADOMSKA-BUGAJ, M. y cols.: "An Estimate of prostate cancer prevalence for a demographically similar workforce population”. W. V. Med. J., 95: 116, 1999.

**2. JOAN AREAL CALAMA: "Biopsia prostática ¿Cuándo y cómo debe plantearse?" Hospital Practice, 4: 53, 2002.

*3. SCHERSTEN, T.; BAIH, M.A.; ASUA, J. y cols.: "Prostate cancer screening. Evidence synthesis and update. Statement of findings". INHATA joint projet. Vitoria-Gasteiz: Office of Health Technology Assesment (Osteba). Department of Health Basque Government. 1999.

*4. NEAL, D.; DONNOVAN, J.: "Prostate Cancer: to screen or not to screen ?" Lancet Oncol, 1: 17, 2000.

5. SMITH, P.G.: "Comparison between registries: agestandardized rates". Parkin D H; Muir C.S; Whelan S.L; Gao Y.T; Ferlay J; Powell J. Cancer incidence in five continents. Vol VI. IARC. Scientific Publications $\mathrm{N}^{\circ}$ 120; Lyon, 1992.

6. Atlas de incidencia de Cáncer en Asturias 1982-1993: mapas municipales y evolución temporal. Consejería de Salud y Servicios Sanitarios y Servicio de publicaciones del Principado de Asturias.

*7. BARRY, M.J.: "Prostate-specific-antigen testing for early diagnosis of prostate cancer". N. Engl. J. Med.,
344: 1373, 2001.

8. CARTER, H.B.; PEARSON, J.D.; METTER, E.J. y cols.: "Longitudinal evaluation of prostate-specific antigen levels in men with and without prostate disease". JAMA, 267: 2215, 1992.

*9. FOWLER, F.J.; McNAUGHTON COLLINS, M.; ALBERTSEN, P. y cols.: "Comparison of recommendations by urologists and radiation oncologists for treatment of clinically localized prostate cancer". JAMA, 283: 3217, 2000.

10. BABAS, S.: "Epidemiology of cancer of the prostate. Analysis of Countries of high and low incidence in prostate cancer". GH Jacobi; R Hohenfellner, cap.2, Williams and Wilkins, Baltimore; 1982.

11. U.S. Preventive Services Task Force, 1999. http://www..Ahcpr.gov.

**12. ALONSO, J.M.; BELLAS, B.; CIERCO, P. y cols.: "Prevención del cáncer". Atención Primaria, 24 (supl.1): 76, 1999.

*13. The NHS Prostate Cancer Programme. http://www.Doh.gov.uk/cancer/pdfs/prostate.pdf.

14. MOLD, J.W.; HOLTGRAVE, D.K.; KISONNI, K.S. y cols.: "The evaluation and treatment of men with asymptomatic prostate nodules in primary care: a decision analysis". J. Fam. Pract., 34: 561, 1992.

*15. KRAHN, M.D.; MAHONEY, J.E.; ECKMAN, M.H. y cols.: "Screening for prostate cancer. A decision analytic view". JAMA, 272: 773, 1994.

16. FLEMING, C.; WASSON, J.H.; ALBERTSEN, P.C. y cols.: "A decision analysis of alternative treatment strategies for clinically localized prostate cancer". JAMA, 269: 2650, 1993.

**17. ROERHBAN, C.G.; PREKENS, C.J.; CARNADY, T.: "Variability of repeated serum PSA measurements within less than 90 days in a well defined patient population”. Urology, 47: 59, 1996.

18. DEJTER, S.W.; MARTIN, J.S.; McPHERSON, R.A. y cols.: "Daily variability in human serum prostate-specific antigen and prostatic acid phosphatase: A comparative evaluation". Urology, 32: 288, 1988.

19. MAATMAN, T.J.: "The role of prostate specific antigen as a tumor marker in men with advanced adenocarcinoma of the prostate". J. Urol., 141: 1378, 1989.

20. RANA, B.; CHSIHOLM, G.B.: "He sold his bike for a low prostate-specific antigen”. J. Urol., 151: 700, 1994.

**21. OESTERLING, J.E.; JACOBSEN, S.J.; CHUTE, C.G. y cols.: "Serum prostate-specific antigen in a community-based population healthy men: establishment of age-specific reference ranges". JAMA, 270: 860, 1993.

22. PARTIN, A.W.; CRILEY, S.R.; SUBONG, E.N.P. y cols.: "Standard versus age-specific prostate specific antigen reference ranges among men with clinically localized prostate cancer: a pathological analysis". J. Urol., 155: 1336, 1996. 